\section{Prueba de basculación (Tilt Table Testing): Comparación de dos protocolos: isoproterenol versus nitroglicerina}

\author{
PEDRO JIMÉNEZ-COHL, SEBASTIÁN VEGA ${ }^{a}$, ROCÍO CÁCERES ${ }^{a}$, \\ CONSTANZA GÓMEZ ${ }^{\text {a }}$ NADIA ROJAS ${ }^{a}$
}

\section{Comparison of the use of isoproterenol or nitroglycerine during the Tilt test}

Background: There is debate about the advantages of different protocols usefulness of tilt test for the diagnosis of vasovagal collapse. Aim: To compare the sensitivity, specificity, adverse reactions, complications and time requirements of two different Tilt test protocols. Material and Methods: A Tilt test using isoproterenol in progressive doses $(2 \mu \mathrm{g}$ for $10 \mathrm{~min}$ and $5 \mu \mathrm{g}$ for $5 \mathrm{~min}$ posteriorly was performed in 159 patients aged 9 to 84 years (59 males). Another Tilt test using sublingual nitroglycerine in doses of $0.3 \mathrm{mg}$ was performed in 201 patients aged 8 to 87 years (62 males). Also, 20 healthy volunteers were tested. Results: The positivity rates of the tests using isoproterenol and nitroglycerin were 75.5 and $77.6 \%$ respectively (NS). The figures for sensitivity were 98.4 and $99.3 \%$ (NS). The figures for specificity were 93.2 and $98.4 \%$ (NS). The test using isoproterenol requires 15 more minutes. As adverse reactions, 38\% of participants experienced palpitations with isoproterenol and 22\% experienced headache with nitroglycerin. Conclusions: The Tilt test with nitroglycerin is shorter, simpler, painless, with less personnel involved and has the same diagnostic accuracy than the test with isoproterenol.

(Rev Med Chile 2015; 143: 69-76)

Key words: Isoproterenol; Nitroglycerin; Primary dysautonomia; Syncope.

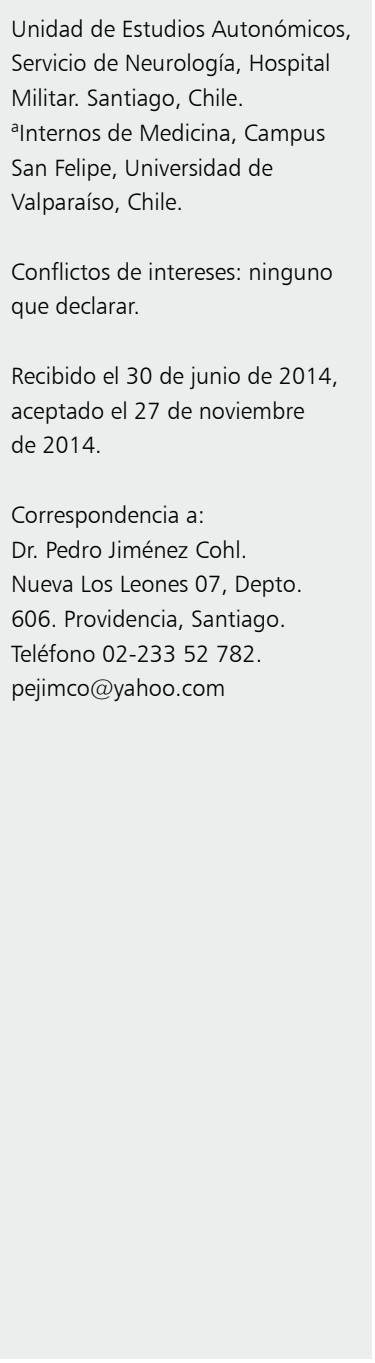

$\mathrm{E}$ n marzo de 2003 publicamos nuestra experiencia en 107 pacientes enviados a una prueba de basculación (“Tilt Test” TT) ${ }^{1}$. Ese protocolo que utilizaba en fase activada isoproterenol (ISO-TT) fue reemplazado por los autores desde 2009 por el uso de nitroglicerina sublingual 0,3 mg (NTG-TT).

El protocolo ISO-TT incluía la colocación de una vía venosa, una bomba de infusión, mayor número de personal involucrado, dolor para el paciente y mayor número de cambios de postura.

También realizamos ambos estudios en 20 voluntarios sanos.

Comparamos ambos protocolos en cuanto a sensibilidad, especificidad, reacciones adversas, riesgos y consumo de tiempo.

\section{Pacientes y Métodos}

Del protocolo ISO-TT, tomamos una muestra de 159 pacientes y del protocolo NTG-TT tomamos 201. Se registraron los datos clínicos de pacientes y controles (Tabla 1).

La edad y sexo de los pacientes se muestran en la Tabla 2.

\section{Estudio previo al Tilt Test}

Evaluación obligatoria por cardiología o neu- 
Tilt Test: isoproterenol versus nitroglicerina - Jiménez-Cohl et al

Tabla 1. Características de los pacientes y controles

\begin{tabular}{|c|c|c|c|c|c|c|c|c|c|c|}
\hline \multirow{2}{*}{$\begin{array}{l}\text { Estudio } \\
\text { ISO-TT Controles }\end{array}$} & \multicolumn{2}{|c|}{ n de casos } & \multicolumn{2}{|c|}{ Varones (n) } & \multicolumn{2}{|c|}{ Mujeres (n) } & \multicolumn{2}{|c|}{$\begin{array}{l}\text { Promedio de } \\
\text { edad (años) }\end{array}$} & \multicolumn{2}{|c|}{$\begin{array}{c}\text { Rango de edad } \\
\text { (años) }\end{array}$} \\
\hline & 159 casos & 20 v. sanos & 59 & 10 & 100 & 10 & 37,6 & 36,5 & $9-84$ & $10-82$ \\
\hline NTG-TT Controles & 201 casos & 20 v. sanos & 62 & 10 & 139 & 10 & 36,5 & 38,7 & $8-87$ & $11-86$ \\
\hline
\end{tabular}

Tabla 2. Pacientes estudiados por edad y sexo

\begin{tabular}{|c|c|c|c|c|c|c|c|c|c|}
\hline \multicolumn{5}{|c|}{ Protocolo ISO Tilt Test } & \multicolumn{5}{|c|}{ Protocolo NTG Tilt Test } \\
\hline \multirow{2}{*}{$\begin{array}{l}\text { Edad } \\
1-9\end{array}$} & \multicolumn{2}{|c|}{ Varones ISO } & \multicolumn{2}{|c|}{ Mujeres ISO } & \multirow{2}{*}{$\begin{array}{l}\text { Edad } \\
1-9\end{array}$} & \multicolumn{2}{|c|}{ Varones NTG } & \multicolumn{2}{|c|}{ Mujeres NTG } \\
\hline & 1 & $(50 \%)$ & 1 & $(50 \%)$ & & 1 & $(50 \%)$ & 1 & $(50 \%)$ \\
\hline $10-19$ & 21 & $(36 \%)$ & 37 & $(64 \%)$ & $10-19$ & 17 & $(28 \%)$ & 44 & $(72 \%)$ \\
\hline $20-29$ & 16 & $(32 \%)$ & 34 & $(68 \%)$ & $20-29$ & 17 & $(30 \%)$ & 39 & $(70 \%)$ \\
\hline 30-39 & 2 & (33\%) & 4 & $(67 \%)$ & $30-39$ & 2 & $(29 \%)$ & 5 & $(71 \%)$ \\
\hline $40-49$ & 2 & $(28 \%)$ & 5 & (72\%) & $40-49$ & 4 & $(31 \%)$ & 9 & $(69 \%)$ \\
\hline $50-59$ & 3 & $(37 \%)$ & 5 & $(63 \%)$ & $50-59$ & 6 & $(35 \%)$ & 11 & $(65 \%)$ \\
\hline $60-69$ & 3 & (37\%) & 5 & $(63 \%)$ & $60-69$ & 2 & $(14 \%)$ & 12 & (86\%) \\
\hline $70-79$ & 5 & $(42 \%)$ & 7 & $(58 \%)$ & $70-79$ & 7 & $(35 \%)$ & 13 & $(65 \%)$ \\
\hline $80-89$ & 6 & $(75 \%)$ & 2 & $(25 \%)$ & $80-89$ & 6 & $(55 \%)$ & 5 & $(45 \%)$ \\
\hline Total: 159 & 59 & $(37 \%)$ & 100 & $(63 \%)$ & Total: 201 & 62 & (31\%) & 139 & $(69 \%)$ \\
\hline
\end{tabular}

rología, incluyendo anamnesis, examen físico, electrocardiograma, ecocardiograma bidimensional y Holter de $24 \mathrm{~h}$. Se indica estudio electrofisiológico cuando existe cardiopatía estructural o sospecha de síncope arrítmico.

\section{Condiciones del examen}

Se realiza en ayuno, entre las 8 y las $12 \mathrm{~h}$, en una habitación tranquila, con luz tenue y temperatura entre 20 y $22^{\circ} \mathrm{C}$. Participan un neurólogo, un cardiólogo y un tecnólogo médico. Personal de cardiología coloca el monitoreo electrocardiográfico continuo.

Para descartar una hipoglicemia, un hemoglucotest es practicado previo al examen.

\section{Protocolos de examen}

a) La prueba de respiración profunda (índice RR); b) La maniobra de Valsalva y c) Examen de Tilt Test (TT).

Se lleva un registro de FC y PA y de síntomas referidos por el paciente cada $5 \mathrm{~min}$. Se anota en cualquier momento el motivo de interrupción del examen o de cualquier incidente importante. El protocolo ISO-TT se fundamenta en Fitzpatrick et $\mathrm{al}^{2}$. El NTG-TT en Del Rosso et $\mathrm{al}^{3}$.

1. ISO-TT: Interrogatorio previo (10 $\mathrm{min}) /$ Instalación cánula braquial (7 min)/Instalación de monitoreo (electrocardiograma continuo y manguito digital para medir la PA) (15 min)/Índice RR y maniobra de Valsalva (10 min)/Instalación de bomba de infusión continua para isoproterenol $(10 \mathrm{~min}) / \mathrm{TT}$ basal (horizontal a $\left.180^{\circ}\right) 10 \mathrm{~min} / \mathrm{TT}$ pasivo (de pie a $70^{\circ}$ ) $20 \mathrm{~min} /$ Descanso acostado por $5 \mathrm{~min} / \mathrm{De}$ pie TT activado con isoproterenol (2 gammas por minuto) por $10 \mathrm{~min} /$ Decúbito dorsal por $5 \mathrm{~min} / \mathrm{De}$ pie (isoproterenol 5 gammas por minuto) por $5 \mathrm{~min} /$ Acostado recuperación final (8 $\mathrm{min}) /$ Retiro de cánula braquial (1 min). Basculación total $45 \mathrm{~min}$. Total aproximado $120 \mathrm{~min}$.

2. NTG-TT: Interrogatorio previo (10 $\mathrm{min}) /$ Instalación de monitoreo (manguito digital para medir PA y electrocardiograma continuo) (15 $\mathrm{min}$ )/Índice RR y maniobra de Valsalva (10 min)/ 
TT basal (horizontal) por $10 \mathrm{~min} / \mathrm{TT}$ pasivo (de pie a $\left.70^{\circ}\right) 45 \mathrm{~min} / \mathrm{TT}$ activado con $0,3 \mathrm{mg}$ de trinitrina sublingual (sin acostar al paciente) por 10 $\mathrm{min} /$ Acostado recuperación final (6 min). Basculación total: $55 \mathrm{~min}$. Total aproximado: $106 \mathrm{~min}$.

La prueba termina si el paciente tiene un "TT positivo": Esto es síncope (pérdida de conciencia) o presíncope (mareos, náuseas, palidez, etc. que anuncian que el síncope es inminente) asociado con hipotensión arterial (PAS $<70 \mathrm{mmHg}$ ) o hipotensión arterial más bradicardia. $\mathrm{O}$ si se producen molestias intolerables para el paciente. Si no hay síntomas se finaliza al término del protocolo.

El equipamiento se compone de: Monitor digital (Ohmeda 2300 Finapres BP Monitor USA). Manguito digital que se coloca en el dedo índice o medio para medir PA y FC en forma continua. Mesa basculante eléctrica (Magnetic Manumed USA) y monitor de electrocardiograma (Quinton Q4500 USA). El paciente es sujetado a la mesa basculante con dos bandas de velcro, una en sus rodillas y otra en el tórax.

El estudio ha sido aprobado por el Comité de Ética del Hospital Militar y se realizó en conformidad con las normas establecidas en la Declaración de Helsinki. Los pacientes y controles dieron su consentimiento informado antes del examen.

\section{Estadística}

Se usa la prueba del $\chi^{24}$ para comparar las variables clínicas y demográficas y las respuestas positivas durante la fase de basculación. En todos los casos, el nivel de confianza fue de $95 \%$. Se consideró como estadísticamente significativo, niveles de $\mathrm{p}<0,05$.

\section{Resultados}

Cerca del $60 \%$ o más de los casos tienen menos de 30 años de edad en ambas casuísticas.
Las lipotimias y síncopes afectan en mayor proporción a jóvenes y en especial a mujeres ${ }^{5,6}$ para luego disminuir su frecuencia por sobre los 30 años.

Durante el TT pasivo, 48 pacientes tuvieron una respuesta positiva (Tabla 3 ) con el protocolo ISO-TT y 76 pacientes con el protocolo NTG-TT $(\mathrm{p}<0,05)$.

En el Tilt Test activado se sumaron 72 pacientes al agregar isoproterenol con el primer protocolo y 80 pacientes al agregar nitroglicerina con el segundo ( $\mathrm{p}>1,00 \mathrm{NS}$ ).

$\mathrm{Al}$ tener una fase pasiva más corta (sólo 20 $\mathrm{min}$ ) en el protocolo ISO TT, se llega a una menor positividad en esa fase. Esa etapa pasiva de $20 \mathrm{~min}$ detectó al 40\% (48/120) de los TT que resultaron positivos, versus el protocolo NTG que con sus 45 min de TT pasivo, detectó al 49\% (76/156) de los casos positivos $(\mathrm{p}<0,05)$.

Con ISO-TT el tiempo promedio necesario para que se manifiesten los síntomas (Tabla 4) fue de 28 min versus 38 min con NTG-TT (p: $0,1 \mathrm{NS}$ ). Eso es porque con ISO TT la fase pasiva era más corta (20 min) y se pasaba rápidamente a usar el activador, de modo que el examen se hacía positivo en menos tiempo.

El síncope vaso vagal con el $45 \%$ es el diagnóstico más frecuente de obtener (Tabla 5). Se divide en 3 variantes fisiopatológicas: Tipos I (Mixta), II (Cardio inhibitoria) y III (vasodepresora). Le sigue en frecuencia la hipotensión ortostática (20\%), que en ambos protocolos cumplió en un tercio de los casos el requisito de ser "precoz" (caída de la PA en los primeros $3 \mathrm{~min}$ ), llevando a síntomas ortostáticos que obligaron a terminar el TT en promedio a los $20 \mathrm{~min}$. En los otros dos tercios, la caída de la PA se debe considerar "tardía" (después de los 3 min en pie). En éstos los síntomas que obligaron a terminar el TT ocurrieron en promedio a los $36 \mathrm{~min}$.

Tabla 3. Positividad del examen de Tilt Test

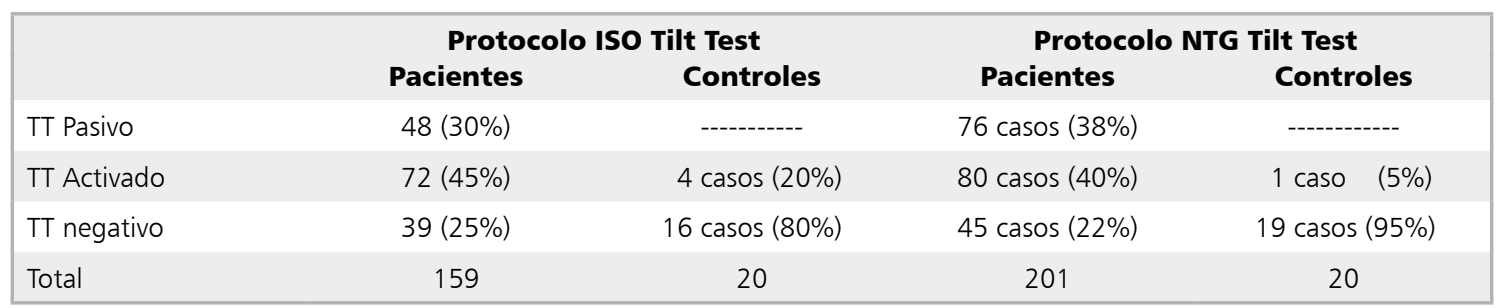


Tabla 4. Tiempo para llegar a TT Positivo (sólo casos con TT positivo)

\begin{tabular}{|c|c|c|c|c|c|}
\hline & \multicolumn{3}{|c|}{ ISO tilt Test ( $\bar{x}: 28$ min) } & \multicolumn{2}{|c|}{ NTG Tilt Test ( $\bar{x}: 38$ min) } \\
\hline & TT Pasivo & $\begin{array}{l}\text { Activado } \\
2 \text { Gammas }\end{array}$ & $\begin{array}{l}\text { Activado } \\
5 \text { Gammas }\end{array}$ & TT Pasivo & $\begin{array}{c}\text { Activado } \\
0,3 \text { NTG }\end{array}$ \\
\hline $\begin{array}{l}\text { Tiempo } \\
\text { n pctes }\end{array}$ & $\begin{array}{c}17 \min (9-20) \\
n=48\end{array}$ & $\begin{array}{c}7 \min (2-10) \\
n=28\end{array}$ & $\begin{array}{c}3,5 \min (1-5) \\
n=44\end{array}$ & $\begin{array}{c}25 \min (1-45) \\
n=76\end{array}$ & $\begin{array}{c}6 \min (1-10) \\
n=80\end{array}$ \\
\hline Controles & ----- & ----- & $\begin{array}{c}4 \min (3-5) \\
n=4\end{array}$ & ----- & $\begin{array}{l}7 \min \\
n=1\end{array}$ \\
\hline
\end{tabular}

Tabla 5. Diagnósticos obtenidos en el Tilt Test

\begin{tabular}{|lrlrc|}
\hline & \multicolumn{2}{c}{$\begin{array}{c}\text { Protocolo } \\
\text { ISO }\end{array}$} & \multicolumn{2}{c|}{$\begin{array}{c}\text { Protocolo } \\
\text { NTG }\end{array}$} \\
\hline S Mixto (tipo I) & 38 & $(24 \%)$ & 47 & $(23 \%)$ \\
S Vasodepresor (Tipo III) & 29 & $(18 \%)$ & 35 & $(18 \%)$ \\
S C Inhibitorio (Tipo II) & 4 & $(2,5 \%)$ & 10 & $(5 \%)$ \\
\hline Hipotortostática & 33 & $(20 \%)$ & 43 & $(21 \%)$ \\
Reac a fármacos & 2 & $(1,2 \%)$ & 3 & $(1,5 \%)$ \\
P.O.T.S. & 7 & $(4 \%)$ & 9 & $(4,5 \%)$ \\
S Post Prandial & 1 & $(0,6 \%)$ & 1 & $(0,5 \%)$ \\
M.S.A. & 1 & $(0,6 \%)$ & 1 & $(0,5 \%)$ \\
S Multifactorial** & 5 & $(3 \%)$ & 8 & $(4 \%)$ \\
Total TT Positivos & 120 & $(75,5 \%)$ & 156 & $(77,6 \%)$ \\
TT Negativo & 39 & $(24,5 \%)$ & 45 & $(22,4 \%)$ \\
Totales & 159 & $(100 \%)$ & 201 & $(100 \%)$ \\
\hline
\end{tabular}

**Sincope multifactorial: Pacientes que tienen una tendencia muy menor ("disautonomía mínima") a tener síncopes vasovagales.

El síndrome de taquicardia ortostática (POTS) alcanza al 4\% de nuestros diagnósticos.

El "síncope multifactorial" (4\%), involucra pacientes que tienen una tendencia muy menor a tener síncopes vasovagales. De hecho tienen o un síncope único, o que se repite muy pocas veces durante su vida, debiendo conjugarse varios factores simultáneamente para que ocurra. Estos casos siempre eran portadores de hiperlaxitud ligamentosa. Existen pacientes que hacen síncopes secundarios al uso de fármacos hipotensores y vasodilatadores $(1,5 \%)$. Se ve principalmente en personas de la tercera edad.

El síncope post prandial es un tipo de "síncope situacional" que se vio en el $0,5 \%$ de nuestros casos y se da luego de alimentarse. Enfermedades
Tabla 6. Efectos adversos/complicaciones en el Tilt Test

\begin{tabular}{|c|c|c|}
\hline & ISO Tilt-Test & NTG Tilt-Test \\
\hline Palpitaciones & 60 (38\%) & $12(6 \%)$ \\
\hline Náuseas-vómitos & $24(15 \%)$ & $6 \quad(3 \%)$ \\
\hline Cefalea & $18(11 \%)$ & $48(24 \%)$ \\
\hline Síncope convulsivo & $4 \quad(2,5 \%)$ & $6(3 \%)$ \\
\hline Asistolía & $2(1,2 \%)$ & $1 \quad(0,5 \%)$ \\
\hline
\end{tabular}

neurológicas poco prevalentes como la atrofia multisistémica (MSA), las encontramos con una frecuencia de $0,5 \%$.

\section{Recuperación post Tilt Test}

Tiempo de recuperación post TT (paciente acostado, con PA y FC recuperadas y sensación de bienestar corporal):

Pacientes ISO: 10,3 min (Rango: 5-20 min).

Controles ISO: 5,2 min (Rango: 3-7 min).

Pacientes NTG: 5 min (Rango 3,5-9 min).

Controles NTG: 5 min (Rango 3-6 min).

Pese a que el promedio de tiempo para recuperarse del ISO-TT supera en $5 \mathrm{~min}$ al del NTG TT, esta diferencia no es significativa ( $\mathrm{p}: 0,15 \mathrm{NS}$ ).

ISO-TT induce mucho más frecuentemente náuseas y vómitos. Por ello el promedio de tiempo para que un paciente se recupere es un poco mayor (Tabla 6).

\section{Efectos adversos}

Palpitaciones y náuseas o vómitos (Tabla 6) fueron infrecuentes con NTG, en relación a su frecuencia con ISO $(\mathrm{p}<0,05)$.

La cefalea, aunque bien tolerada, es un poco más del doble al usar NTG $(\mathrm{p}<0,05)$. Pocas veces persiste por 2 ó 3 min después del examen. 
"Síncope convulsivo" como evento final, resultó prácticamente igual en ambos protocolos. Su aparición se relacionó con la presencia de una respuesta cardioinhibitoria, en la que bajan la PA y FC en forma tan intensa que se producen mioclonías secundarias a la isquemia cortical.

Con ISO, en 2 casos llegamos a la asistolia, en la fase activada con 5 gammas. Una duró 4 seg y otra 6 seg. Con NTG-TT, sólo tuvimos una asistolía de 5 seg durante la fase activada. Todas revertieron en menos de siete segundos en posición de Trendelenburg.

\section{Discusión}

La validez diagnóstica de un examen de TT está en debate, no existiendo un "Gold Standard"7-9. En nuestra experiencia hemos acordado que si: a) Los síntomas del paciente durante el TT y b) Los hallazgos hemodinámicos obtenidos en el TT son concordantes con el motivo de consulta del paciente, entonces el valor del examen debe ser correcto.

Esta correlación de síntomas que acontecen durante un TT, suele ser bastante buena con lo que le ocurre al paciente en la vida real. En el protocolo ISO TT el año $2003^{1}$ sólo $15 \%$ de los pacientes dijeron que la sintomatología durante la fase activada fue más intensa o bien apareció muy precozmente con respecto a la que presentaban ellos en la vida real. Con NTG-Tilt Test todos los pacientes han correlacionado correctamente sus síntomas durante el TT, con lo que les ocurre en la vida real.

La fase activada es importante para la positividad del examen, ya que aporta un importante número de casos positivos. Se suma el $45 \%$ de casos en el protocolo ISO-TT y el $40 \%$ en el NTGTT. De no usarse se pasarían por alto un gran número de casos que teniendo un cuadro clínico muy indicador de disautonomía, tienen un TT pasivo negativo.

Para conocer la frecuencia de falsos positivos, citamos a TT a controles sanos. No tuvimos controles que fuesen positivos, durante la fase pasiva del TT en ninguno de los 2 protocolos, pero sí en la fase activada. El NTG-TT muestra en voluntarios sanos 5\% de "falsos positivos", contra 20\% del protocolo ISO-TT $(\mathrm{p}<0,05)$. Este número sólo apareció con el uso de 5 gammas de isoproterenol. Por ello este protocolo fue abandonado, quedándonos desde el año 2003 al 2009 con sólo 3 gammas de isoproterenol en la fase activada. En el año 2009 cambiamos definitivamente al protocolo NTG-TT.

\section{Edad y sexo}

A toda edad y con ambos protocolos (excepto después de los 80 años) el predominio del sexo femenino es notorio ${ }^{5,6}$. Se postula que se debe a la mayor frecuencia de hiperlaxitud ligamentosa en las mujeres ${ }^{10-12}$, lo que las predispone a los síncopes. Después de los 40 años y hasta los 69, se puede ver una moderada caída en los resultados positivos. Esto se debería a la disminución (relativa) en la frecuencia de síncopes en esas edades ${ }^{10-12}$. Esta frecuencia vuelve a subir después de los 70 años debido a la aparición de hipotensión ortostática, cardiopatías orgánicas, neuropatías autonómicas y enfermedades degenerativas del sistema nervioso autónomo ${ }^{5,6,11,13}$.

\section{Sensibilidad y especificidad}

La positividad global del TT es mayor al $75 \%$ en ambos protocolos (Tabla 7), sin mayor diferencia (ISO-TT: 75,5\% vs NTG-TT: 77,6\%) (p: 0,220 NS) entre ambos. Así, a lo menos por su positividad, daría igual usar uno u otro.

Es importante la selección de los casos estudiados. Todo paciente debe ser derivado al examen con estudio previo y realizarles un electroencefalograma y un Holter de ritmo cardíaco antes del TT, para descartar 2 causas muy frecuentes de diagnóstico diferencial: epilepsia y arritmia ${ }^{7}$.

La sensibilidad para el protocolo ISO-TT resultó ser 98,4\% y para NTG-TT 99,3\%, diferencia no significativa (p: 2,20 NS). La especificidad para ISO-TT con 5 gammas resultó ser 93,2\% y para NTG-TT 98,4\%, diferencia no significativa (p: 2,05 NS).

Aunque sensibilidad y especificidad resultaron muy similares entre ambos, la especificidad fue algo mejor con el protocolo NTG-TT, porque ISO-TT con 5 gammas arroja más falsos positivos.

Comparaciones entre ambos protocolos muestran que la activación con nitroglicerina o isoproterenol mejora la sensibilidad de un TT pasivo $^{5,15,16,17-22}$. Incluso describen una sensibilidad y especificidad muy similares para isoproterenol y nitroglicerina ${ }^{5,17-22}$. Un estudio mostró algo similar al nuestro, que ISO con altas dosis es menos específico que $\mathrm{NTG}^{23}$. 
Tilt Test: isoproterenol versus nitroglicerina - Jiménez-Cohl et al

Tabla 7. Positividad del Tilt Test según edad

\begin{tabular}{|c|c|c|c|c|c|c|c|c|}
\hline \multirow{3}{*}{$\begin{array}{l}\text { Edad } \\
\text { Años } \\
1-9\end{array}$} & \multicolumn{4}{|c|}{ Protocolo ISO Tilt Test } & \multicolumn{4}{|c|}{ Protocolo NTG Tilt Test } \\
\hline & \multicolumn{2}{|c|}{$\begin{array}{l}\text { n de pacientes } \\
\text { por edad }\end{array}$} & \multicolumn{2}{|c|}{$\begin{array}{c}\text { n casos } \% \text { con } \\
\text { TT Positivo }\end{array}$} & \multicolumn{2}{|c|}{$\begin{array}{l}\text { n de pacientes } \\
\text { por edad }\end{array}$} & \multicolumn{2}{|c|}{$\begin{array}{l}\text { n casos } \% \text { con } \\
\text { TT Positivo }\end{array}$} \\
\hline & 2 & $1,2 \%$ & 2 & $100 \%$ & 2 & $0,9 \%$ & 2 & $100 \%$ \\
\hline $10-19$ & 58 & $36 \%$ & 46 & $79 \%$ & 61 & $30,3 \%$ & 49 & $80 \%$ \\
\hline $20-29$ & 50 & $31 \%$ & 37 & $74 \%$ & 56 & $27,8 \%$ & 41 & $73 \%$ \\
\hline $30-39$ & 6 & $3,7 \%$ & 5 & $83 \%$ & 7 & $3,4 \%$ & 7 & $100 \%$ \\
\hline $40-49$ & 7 & $4,4 \%$ & 4 & $57 \%$ & 13 & $6,4 \%$ & 8 & $61 \%$ \\
\hline $50-59$ & 8 & $5,0 \%$ & 6 & $75 \%$ & 17 & $8,4 \%$ & 13 & $76 \%$ \\
\hline $60-69$ & 8 & $5,0 \%$ & 5 & $62 \%$ & 14 & $6,9 \%$ & 10 & $71 \%$ \\
\hline 70-79 & 12 & $7,5 \%$ & 9 & $75 \%$ & 20 & $9,9 \%$ & 16 & $80 \%$ \\
\hline $80-89$ & 8 & $5,0 \%$ & 6 & $75 \%$ & 11 & $5,4 \%$ & 10 & $91 \%$ \\
\hline Total & \multicolumn{2}{|c|}{159 casos: $100 \%$} & 120 & $(75,5 \%)$ & \multicolumn{2}{|c|}{201 casos: $100 \%$} & 156 & $(77,6 \%)$ \\
\hline
\end{tabular}

\section{Complicaciones y efectos adversos}

El TT resultó un examen seguro, con muy pocas complicaciones. Como reacciones adversas fueron comunes molestias menores: palpitaciones (38\%) o náuseas y vómitos (15\%) con isoproterenol o cefalea $(22 \%)$, con la nitroglicerina. Otros autores reportan similar experiencia ${ }^{17-22}$.

Algunos pacientes presentaron asistolías durante el examen (Tabla 6), pero éstas no pueden considerarse una complicación sino más bien un objetivo o punto final del TT.

Llevar al paciente a la posición de decúbito dorsal y/o Trendelenburg fue suficiente para normalizar la frecuencia cardíaca y presión arterial $^{24-26}$. Con isoproterenol se han reportado asistolías de más de $20 \operatorname{seg}^{21,27-29}$ y bloqueo atrioventricular que requirió de atropina ${ }^{17}$.

Con nitroglicerina en "spray" sublingual 400 $\mathrm{mg}^{17,29}$ se han reportado asistolías, en niños y adolescentes bajo los 18 años de edad y peso corporal menor a $50 \mathrm{Kg}^{17-29}$. Este grupo tendería a hacer más cardioinhibición (20-28\% de las respuestas positivas) durante el TT activado, ya sea con isoproterenol o con nitroglicerina ${ }^{17-29}$.

En nuestros pacientes no tuvimos arritmias ni angina, con ninguno de los dos protocolos. La presentación de arritmia completa por fibrilación auricular ${ }^{22,28-30}$ puede ocurrir durante o posterior a la prueba de basculación (Tilt Table Testing). Generalmente es autolimitada. Se han documentado arritmias ventriculares de potencial riesgo vital con isoproterenol, en presencia de cardiopatía isquémica o enfermedad del nódulo sinusal ${ }^{27,28,31}$. No se han publicado estas complicaciones ${ }^{22,30} \mathrm{con}$ el uso de nitroglicerina.

\section{Duración de la prueba}

El protocolo ISO TT, al involucrar la colocación de una vía venosa y de una bomba de infusión, eleva el tiempo del examen en unos $15 \mathrm{~min}$. Si a esto sumamos un período de recuperación post examen un poco mayor, se llega a una duración de dos horas. Si se dan problemas técnicos con la bomba de infusión o la vía venosa se puede demorar aún más. Eso no sucede con nitroglicerina sublingual.

La vía venosa es molesta para los pacientes, incluso en algunos la punción puede provocar sensación de lipotimia por respuesta vasovagal aumentada a este estímulo, por lo que se recomienda realizar con el paciente en decúbito dorsal.

\section{Conclusiones}

El protocolo NTG-TT en nuestra serie presentó positividad de 78\% comparado con $75 \%$ del protocolo ISO-TT, sin diferencias estadísticas significativas de sensibilidad y especificidad.

El protocolo NTG-TT presenta ventajas debido a la menor duración de la prueba y no ser necesaria la punción venosa para administrar medicación 
Tilt Test: isoproterenol versus nitroglicerina - Jiménez-Cohl et al

intravenosa. Además sus efectos adversos descritos son leves (cefalea) y se requiere de menos personal de apoyo.

Agradecimientos: Al personal encargado de colocar el monitoreo electrocardiográfico: Mónica San Martín, Carmen Vidal, Carmen Navarro, Erica Collío, Paulina Palomo y Minerva Jara. Las enfermeras del Servicio de Cardiología fueron: Ana Castro, Leonor Acevedo y Clotilde Sanhueza. El Dr. Beltrán González colaboró en este estudio desde 1996 hasta su sensible fallecimiento en abril de 2010.

\section{Referencias}

1. Jiménez-Cohl P, González B, Cugniet O, Thieck E. Utilidad diagnóstica del Tilt Test. Rev Chil Epilepsia 2003; 4 (1): 20-3.

2. Fitzpatrick A, Theidorakis G, Vardas P, Sutton R. Methodology of Head-Up Tilt Testing in Patients With Unexplained Syncope. J Am Coll Cardiol 1991; 17: 12530 .

3. Del Rosso A, Bartoli P, Bartoletti A, Brandinelli-Geri A, Bonechi F, Maioli M, et al. Shortened head-up tilt testing potentiated with sublingual nitroglycerin in patients with unexplained syncope. Am Heart J 1998; 135 (4): 564-70.

4. Prueba chi-cuadrado. http://es.slideshare.net/armando310388/prueba-chicuadrado.

5. Colman N, Nahm K, Ganzeboom KS, Shen WK, Reitsma JB, Linzer M, et al. Epidemiology of reflex syncope. Clin Auton Res (2004) 14 (Suppl 1): 1003-3.

6. Ganzeboom KS, Colman N, Reitsma JB, Shen WK, Wieling W. Prevalence and triggers of syncope in medical students. Am J Cardiol 2003; 91: 1006-8.

7. Grubb B. Neurocardiogenic syncope. N Engl J Med 2005; 352: 1004-10.

8. Forleo C, Guida P, Iacoviello M, Resta M, Monitillo F, Sorrentino S, et al. Head-up tilt testing for diagnosing vasovagal syncope: A meta- analysis. Int J Cardiol 2013; 168 (1): 27-35.

9. Grubb B. Neurocardiogenic syncope and related disorders of orthostatic intolerance. Circulation 2005; 111: 2997-3006.

10. Bravo JF, Wolff C. Clinical study of hereditary disorders of connective tissues in a Chilean population: Joint hypermobility syndrome and vascular Ehlers-Danlos syndrome. Arthritis Rheum 2006; 54 (2): 515-23.

11. Bravo JF, Sanhueza G, Hakim A. Cardiovascular auto- nomic dysfunction and chronic fatigue in fibromyalgia and joint hypermobility syndrome. En Hakim, Keer \& Grahame editors. Hypermobility, Fibromyalgia and Chronic Pain. London UK, Elsevier 2010. Cap 6i.

12. Grahame R. What is the Joint Hypermobility Syndrome? JHS from the cradle to the grave. En Hakim, Keer y Grahame editors. London, UK. Elsevier, 2010, Cap 2. With contributions from Jaime F Bravo, Nathan Hasson, Rosemary Keer \& Susan M Maillard.

13. Sheldon RS, Sheldon AG, Connolly SJ, Morillo CA, Klingenheben T, Krahn AD, Koshman ML, Ritchie D. Investigators of the Syncope Symptom Study and the Prevention of Syncope Trial. Age of first faint in patients with vasovagal syncope. J Cardiovasc Electrophysiol 2006; 17 (1): 49-54.

14. Maw Pin Tan, B Med Sci, BMBS, MRCP, Steve Parry. Vasovagal Syncope in the Older Patient. The American College of Cardiology's Cardiosource 2008. Disponible en: http://www.medscape.com/viewarticle/577022_1/ [Consultado el 21 de abril de 2013].

15. E. Shen WK, Jahangir A, Beinborn D, Lohse CM, Hodge DO, Rea RF, et al. Utility of a single-stage isoproterenol tilt table test in adults. A randomized comparison with passive head-up tilt. J Am Coll Cardiol 1999; 33 (4): 98590.

16. Raybaud F, Camous JP, Jung M, Dadoun M, Baudouy M, Morand P. Comparative data of tilt test with or without isoproterenol in investigation of unexplained unconsciousness and syncope. Arch Mal Coeur Vaiss 1996 Sep; 89 (9): 1145-51.

17. Vlahos AP, Tzoufi M, Katsouras CS, Barka T, Sionti I, Michalis LK, et al. Provocation of neurocardiogenic syncope during head-up tilt testing in children: comparison between isoproterenol and nitroglycerin. Pediatrics 2007; 119 (2): e419-25. Epub 2007 Jan 15.

18. Hermosillo AG, Marquez MF, Jauregui-Renaud K, Falcon JC, Casanova JM, Guevara M, et al. Tilt testing in neurocardiogenic syncope: isosorbide versus isoproterenol. Acta Cardiol 2000; 55 (6): 351-5.

19. Aslan O, Guneri S, Badak O, Kirimli O, Goldeli O, Keskin $\mathrm{V}$, et al. Head-up tilt table testing with low dose sublingual isosorbide dinitrate in the evaluation of unexplained syncope: a comparison with isoproterenol infusion. Can J Cardiol 2002; 18 (8): 853-9.

20. Delépine S, Prunier F, Lefthériotis G, Dupuis J, Vielle B, Geslin $\mathrm{P}$, et al. Comparison between isoproterenol and nitroglycerin sensitized head-upright tilt in patients with unexplained syncope and negative or positive passive head-up tilt response. Am J Cardiol 2002; 90 (5): 488-91.

21. Kapoor WN, Smith MA, Miller NL. Upright tilt testing 
in evaluating syncope: a comprehensive literature review. Am J Med 1994; 97: 78-88.

22. Aerts AJ, Dentale P. Nitrate stimulated tilt table testing: a review of the literature. Pacing Clin Electrophysiol 2003; 26: 1528-37.

23. Natale A, Sra J, Akhtar M, Kusmirek L, Tomassoni G, Leonelli F, et al. Use of Sublingual Nitroglycerin During Head-up Tilt-Table 'Testing in Patients $>60$ Years of Age. Am J Cardiol 1998; 82: 1210-3.

24. Barón-Esquivias G, Martínez-Rubio A. Tilt Table Test State of the art. IPEJ 3 (4); 2003: 239-52.

25. Barón-Esquivias G, Pedrote A, Cayuela A, Valle JI, Fernández JM, Arana E, et al. Longterm outcome of patients with asystole induced by head-up tilt test. Eur Heart J 2002; 23: 483-9.

26. Grubb BP, Kosinski D. Neurocardiogenic syncope and related syndromes of orthostatic intolerance. Cardiology Review 1997; 5 (4): 182-9.

27. Kim PH, Ahn SJ, Kim JS. Frequency of arrhythmic events during head-up tilt in patients with suspected neurocardiogenic syncope or presyncope. Am J Cardiol 2004; 94: 1491-5.

28. Wang $\mathrm{CH}$, Hung MJ, Kuo LT. Cardiopulmonary resuscitation during coronary vasospasm induced by tilt table testing. Pacing Clin Electrophysiol 2000; 23: 2138-40.

29. Swissa M, Epstein M, Paz O, Shimoni S, Caspi A. Headup tilt table testing in syncope: Safety and efficiency of isosorbide versus isoproterenol in pediatric population. Am Heart J 2008; 156 (3): 477-82.

30. Parry SW, Reeve P, Lawson J, Shaw FE, Davison J, Norton M, et al. The Newcastle protocols 2008: an update on headup tilt table testing and the management of vasovagal syncope and related disorders. Heart 2009; 95 : 416-20.

31. Goolamali SI, Loh VL, Sopher M. The head-up tilt test as a cause of myocardial infarction. Europace 2004; 6: 548-51. 